\title{
Molecular characterization of human Cryptosporidium spp. isolates after an unusual increase in late summer 2012
}

Jeroen H. Roelfsema ${ }^{*}$, Hein Sprong ${ }^{1}$, Simone M. Cacciò ${ }^{2}$, Katsuhisa Takumi ${ }^{1}$, Michiel Kroes ${ }^{1}$, Wilfrid van Pelt ${ }^{1}$, Laetitia M. Kortbeek ${ }^{1}$ and Joke W. B. van der Giessen ${ }^{1}$

\begin{abstract}
Background: During the late summer 2012, a number of medical microbiological laboratories (MMLs) reported an unusual increase in cases of cryptosporidiosis, a gastrointestinal infection caused by the protozoan parasites Cryptosporidium spp. Prompted by this signal, the National Institute of Public Health and the Environment (RIVM) started an epidemiological investigation into possible causes. Simultaneously, samples diagnosed at MMLs were sent to RIVM for genotyping, aiming to further identify the possible source of the increase.

Methods: Genotyping was performed by sequencing a fragment of the GP60 gene. Additional genotyping was performed on a subset of samples using six microsatellite markers. Population genetic analysis was performed using BEAST.

Results: The majority of the samples were typed as C. hominis, and a single GP60 genotype (IbA10G2) largely predominated. Genotyping microsatellite markers further supported the circulation of a single genetic type. Population genetic analysis with genotypes found in previous years is inconsistent with a decrease in effective population size.
\end{abstract}

Conclusions: The conclusion of this finding is that the rise reflects more an overall increase and not a common source outbreak.

Keywords: Cryptosporidium, Parasite, GP60, Genotyping, Gastroenteritis, Population genetics

\section{Background}

Cryptosporidium spp. are protozoan parasites and are widespread in a diverse range of hosts such as mammals including humans, birds and fish. In the host Cryptosporidium spp. can cause gastro-enteritis with symptoms of diarrhoea, abdominal pain, nausea and fever [1]. Because of the global distribution of Cryptosporidium spp., these parasites can infect humans everywhere around the world.

There are more than twenty recognized species of Cryptosporidium [2,3]. These species differ remarkably in their host specificity. Indeed, some species have a strong preference for a limited range of hosts, such as $C$. hominis, found

\footnotetext{
* Correspondence: jeroen.roelfsema@rivm.nl

${ }^{1}$ Centre for Infectious Disease Control Netherlands, National Institute for Public Health and the Environment (RIVM), Bilthoven, The Netherlands Full list of author information is available at the end of the article
}

almost exclusively in humans, whereas others, such as $C$. ubiquitum will infect a very broad range of hosts including rodents, cattle and humans $[4,5]$. Humans are mostly infected by $C$. parvum and $C$. hominis [6-9]. C. hominis was first recognized as a distinct strain of $C$. parvum, only found in humans. Other $C$. parvum strains are not restricted to human hosts and can also be found in cattle and other animals $[10,11]$. Later, C. hominis was recognized as a distinct species [4].

Human cryptosporidiosis in Europe occurs with seasonal peaks, particularly in August and September [12]. Seasonal peaks in The Netherlands have been reported: an increase of cryptosporidiosis in the spring, mainly caused by $C$. parvum and a peak late in the summer and the start of the autumn, caused both by $C$. parvum and C. hominis [7]. Detailed reports of seasonal peaks in the UK have been published [13-15]. Similar patterns have 
been described in other parts of the world as well, for instance in California (USA) and New Zealand [16, 17].

Next to sporadic cases, outbreaks of cryptosporidiosis can occur, often through contact with contaminated water containing infectious oocysts. Outbreaks of cryptosporidiosis as a result from a single source contamination occur occasionally in western European countries. Such outbreaks have recently been reported in Norway, Sweden and Spain [18-20]. Typical sources can be food, contaminated swimming pools or recreational water. Water-borne outbreaks are frequently reported worldwide [21, 22].

In 2012 a number of medical microbiological laboratories (MMLs) reported an unusually high increase of cases of cryptosporidiosis starting in August. A rise of cryptosporidiosis in August is to be expected but the diagnostic laboratories reported an increase beyond the ordinary. Based on collected data from eight laboratories that used the same detection method since 2010, a rise of more than three times of the number of patients compared to previous years was observed [23]. The National Institute of Public Health and the Environment (RIVM) also started a case-control study to identify possible sources of Cryptosporidium contamination but found no evidence of a single source outbreak [23]. Additionally, the MMLs sent in the Cryptosporidium-positive samples for genotyping, intended to assist the epidemiological investigation. Here, we present the results of a molecular characterization of the Cryptosporidium spp. isolates during that late summer increase.

\section{Methods}

\section{Sample collection}

Eighteen MMLs sent in Cryptosporidium spp.-positive samples, starting in August 2012. The majority were collected in August but some samples were already collected in July. Sample collection continued until the beginning of December. These samples were sent to the RIVM as either stool or DNA extracts. In case stool samples were received, we isolated DNA using the High Pure PCR template DNA isolation kit from Roche (Almere, The Netherlands) according to the manufacturer's instructions.

\section{Species determination}

We performed a real-time duplex PCR with dual labeled probes on a Roche LightCycler 480 apparatus to determine whether the species was C. parvum or C. hominis. We used a combination of a PCR on C. parvum, developed by Hadfield et al. [24] and a PCR we developed specifically for C. hominis. The C. parvum specific PCR targets a gene for a hypothetical protein and the $C$. hominis PCR targets part of the GP60 gene. The primers and probes are listed in Table 1. Each $25 \mu \mathrm{l}$ reaction contained 10 picomoles (pmol) of each C. parvum primer, CRULib13F and CRULib13RCp
Table 1 List of primers and probes used in this study. Primers AL3531 and AL3535 are used in the first round and primers AL3532 and AL3534 are nested primers used in the second round to amplify part of the GP60 gene. The primers CRULib13F and CRULib13RCp in combination with probe CRULib13TMCp amplify and detect specifically a hypothetical gene of C. parvum whereas primers ChomGP60f, ChomGP60r and probe ChomGP60Tp amplify part of the GP60 and specifically detect C. hominis

\begin{tabular}{llll}
\hline Name & Sequence & 5'label & 3'label \\
\hline ATGFOR & atgagattgtcgctcattatc & & \\
AL3533REV & agatatatcttggtgcg & & \\
AL3531 & atagtctccgctgtattc & \\
AL3535 & ggaaggaacgatgtatct & \\
AL3532 & tccgctgtattctcagcc & & \\
AL3534 & gcagaggaaccagcatc & & \\
CRULib13F & tccttgaaatgaatatttgtgactcg & & \\
CRULib13RCp & ttaatgtggtagttgcggttgaac & & \\
CRULib13TMCp & tatctcttcgtagcggcgta & Vic & MGB-NFQ \\
ChomGP60F & aaagaacaatgaagaaagccaaa & & \\
ChomGP60R & ggtagaaggttgggtagcactct & & \\
ChomGP60Tp & tcaaggtggctccaaaggagacg & Texas Red & BHQ2 \\
\hline
\end{tabular}

and 7.5 pmol of probe CRULib13TMCp, 15 pmol of each C. hominis primer, ChomGP60F and ChomGP60R and 10 pmole probe ChomGP60Tp. We used the LightCycler Taqman master kit from Roche. Amplification took $45 \mathrm{cy}$ cles with $10 \mathrm{~s}$ denaturing at $95{ }^{\circ} \mathrm{C}, 20 \mathrm{~s}$ annealing at $60{ }^{\circ} \mathrm{C}$ and $20 \mathrm{~s}$ extension at $72{ }^{\circ} \mathrm{C}$.

\section{GP60 genotyping}

Genotyping was performed by sequencing a fragment of the GP60 gene. We amplified a fragment of approximately 500 bp using primers ATGFOR and AL3533REV [25]. We also analysed 22 samples using a nested PCR with primers AL3531 and AL3535 for the first round and AL3532 and AL3534 for the second round leading to a fragment of approximately 850 bp [26]. Primers are listed in Table 1. PCRs were performed using Qiagen HotStarTaq (Qiagen, Venlo, The Netherlands). The products were analyzed on a $1.5 \%$ agarose gel, visualized by GelRed Nucleic Acid Gel Stain from Biotium (Hayward, CA, USA). PCR products were treated with ExoSAP-IT from USB (Cleveland, USA) according to the manufacturer's instructions. We sequenced the PCR products on a 3700 automated sequencer from Applied Biosystems (Nieuwerkerk a/d IJsel, The Netherlands), using the Big Dye Terminator kit according to the manufacturer's instructions. 


\section{Microsatellite analysis}

To further investigate genetic variability of parasite isolates, six micro- and mini-satellite markers were analyzed. These markers correspond to the MS1, MS9, TP14, MM5, MM18, and MM19 loci. The MS1 marker contains a GGTGGTATGCCA repeat in the heat shock protein 70 gene located on chromosome 2. The MS9 marker contains a TGGACT repeat in a 2016 bp gene encoding a hypothetical protein located on chromosome 5 . The TP14 marker contains a CAA repeat in a 8421 bp gene encoding a hypothetical protein located on chromosome 8. The MM5 marker contains a TCCTCCTCT repeat within a 11,418 bp gene located on chromosome 6. The MM18 marker contains a GGACCA repeat in a 5004 bp gene, also located on chromosome 8. The MM19 marker contains a GGAGCT repeat in a 7230 bp gene, again located on chromosome 8 . PCR was performed as previously described [27]. The size of each PCR product was estimated by electrophoresis on a capillary apparatus (QiaXcel; Qiagen, Milan, Italy) by comparison to size standards. Each allele was assigned a unique number indicating the estimated size in base pairs.

\section{Estimating genetic diversity}

The genetic diversity of a certain species can be used as an estimate for its effective population size [28]. Spatial or temporal changes in the population size of an infectious agent may reflect the dynamics of the prevalence of the disease (23382432, 23244453, takumi submitted 2015). DNA sequences of GP60 from two different time points were aligned using the multiple alignment software MAFFT [29]. Subsequently, the alignments were analyzed by a molecular evolution approach using the software Bayesian Evolutionary Analysis by Sampling Trees (BEAST) [28]. We set Hasegawa-KishinoYamamoto (HKY) model of DNA evolution and a constant mutation rate for each site of the GP60 sequences. Each simulation took 1,000,000 iterations. We discarded $10 \%$ burn-in and used the rest of posterior samples for the constant population size as a quantification of the genetic diversity. A criterion for a satisfactory convergence for this parameter was an effective sample size greater than 200.

We tested whether the DNA sequences sampled in 2012 indicate a change in the estimated genetic diversity of $C$. hominis compared to the previous years. For this, we subtracted the posterior samples for the constant population sizes based on the sequences collected between 2003 and 2005 [7] from the posterior samples based on the sequences collected in 2012 for the current study. Proportion of positive differences is an estimate for the probability that the genetic diversity of $C$. hominis increased in 2012 compared to the previous years [30].

\section{Results}

Origin of samples

The unusually high increase of cryptosporidiosis was reported by MMLs in The Netherlands. Together, these laboratories cover most of The Netherlands. A total of 507 samples were received and analysed. Table 2 shows the origin of samples.

\section{Species identification by real-time PCR}

Using a duplex real-time PCR, we determined whether the samples contained C. hominis or C. parvum. Out of the 507 samples tested, $410(81 \%)$ could be typed at the species level, of which 360 (88\%) were C. hominis and 50 (12\%) were C. parvum.

\section{Genotyping the samples using GP60 sequences and microsatellite markers}

First, genotyping of the Cryptosporidium samples was performed using the GP60 gene. Approximately half of the 507 samples $(n=269)$ were typed. Genotypes are listed in Table 3 . Nearly all samples, $90 \%(n=243)$, were C. hominis, which is concordant with the results from the real-time PCR assay. The predominant $C$. hominis genotype was IbA10G2, found in $81 \%(n=218)$. In total, 16 different genotypes were found, and most of these were represented by only one or two samples (Table 3 ).

Table 2 Overview of samples sent to the RIVM. The samples came from different regions of The Netherlands. The middle and western part of The Netherlands is highly urbanised

\begin{tabular}{|c|c|c|c|c|c|c|}
\hline Region & Samples & Typed & C. hominis & C. parvum & $\mathrm{IbA} 10 \mathrm{G} 2$ & Other \\
\hline North West & 71 & $46(100 \%)$ & $44(96 \%)$ & $2(4 \%)$ & 36 (78 \%) & $10(22 \%)$ \\
\hline West & 29 & 15 (100\%) & 14 (93\%) & 1 (7 \%) & 14 (93\%) & 1 (7 \%) \\
\hline Middle & 138 & 65 (100\%) & 59 (91 \%) & 6 (9 \%) & 53 (82\%) & 12 (18\%) \\
\hline South & 89 & 55 (100\%) & 51 (93\%) & 4 (7 \%) & 47 (85 \%) & 8 (15 \%) \\
\hline South East & 115 & $42(100 \%)$ & 33 (79 \%) & 9 (21\%) & 30 (71 \%) & 12 (29\%) \\
\hline East & 34 & 25 (100\%) & 23 (92 \%) & 2 (8 \%) & 22 (88 \%) & $4(16 \%)$ \\
\hline North East & 31 & $21(100 \%)$ & 19 (90 \%) & 2 (10\%) & 16 (76 \%) & $5(24 \%)$ \\
\hline Total & 507 & 269 (100\%) & $243(90 \%)$ & 26 (10 \%) & 218 (81 \%) & $52(19 \%)$ \\
\hline
\end{tabular}


Table 3 GP60 genotypes found in isolates from the present study and from 2003 and 2005 [7]

\begin{tabular}{|c|c|c|c|c|c|}
\hline \multirow[t]{2}{*}{ Species } & \multirow[t]{2}{*}{ GP60 genotype } & \multicolumn{2}{|l|}{2012} & \multicolumn{2}{|c|}{ 2003-2005 (Wielinga et al.) } \\
\hline & & Number & Percentage & Number & Percentage \\
\hline C. hominis & $\mathrm{IbA} 10 \mathrm{G} 2$ & 218 & 81.04 & 65 & 82.28 \\
\hline C. hominis & laA14R3 & 21 & 7.81 & & 0 \\
\hline C. hominis & leA11G3T3 & 3 & 1.12 & & 0 \\
\hline C. hominis & laA15 & 1 & 0.37 & & 0 \\
\hline C. hominis & laA24G1R1 & 1 & 0.37 & & 0 \\
\hline C. hominis & IfA12G1 & 1 & 0.37 & & 0 \\
\hline C. hominis & ICA5G3R2 & 0 & 0 & 1 & 1,27 \\
\hline C. hominis & $\mid \mathrm{dA} 14$ & 0 & 0 & 1 & 1,27 \\
\hline C. hominis & $\mid d A 17$ & 0 & 0 & 1 & 1,27 \\
\hline C. parvum & IlaA15G2R1 & 11 & 4.09 & 8 & 10,13 \\
\hline C. parvum & IlaA17G2R1 & 3 & 1.12 & & 0 \\
\hline C. parvum & IlaA17G1R1 & 2 & 0.74 & 1 & 1,27 \\
\hline C. parvum & IICA5G3 & 2 & 0.74 & & 0 \\
\hline C. parvum & $\| d A 17 G 1$ & 2 & 0,74 & & 0 \\
\hline C. parvum & IlaA16G3R1 & 1 & 0,37 & & 0 \\
\hline C. parvum & IIdA18G1 & 1 & 0,37 & 1 & 1,27 \\
\hline C. parvum & IIA19G1 & 1 & 0,37 & & 0 \\
\hline C. parvum & IIdA22G1 & 1 & 0.37 & & 0 \\
\hline C. parvum & IIA15G1 & 0 & 0 & & 0 \\
\hline C. parvum & IIA16G1 & 0 & 0 & 1 & 1,27 \\
\hline
\end{tabular}

The two most common genotypes after IbA10G2 were the $C$. hominis type IaA15 $(n=21)$ and the $C$. parvum type IIaA15G2R1 $(n=11)$.

The unusually high increase of cryptosporidiosis was reported by microbiological diagnostic laboratories that cover most of The Netherlands. When the geographical origin of the different genotypes was analysed, little difference between the various regions was found (Table 2). The increase of cases was observed throughout the country, and the IbA10G2 genotype predominated in all regions.

A panel of 20 randomly selected samples with the IbA10G2 genotype was further genotyped at six microsatellite markers [27]. Out of the 20 samples, 8 were typed at all markers, 8 were typed at most but not all markers, and 4 yielded no amplification. At each of the markers tested, only one allele was found, with estimated size of $374 \mathrm{bp}$ (locus MS1), $261 \mathrm{bp}$ (locus MS9), $261 \mathrm{bp}$ (locus TP14), 210 bp (locus MM5), 233 bp (locus MM18) and 224 bp (locus MM19). Therefore, a single multi-locus genotype (MLG) characterized these $16 \mathrm{C}$. hominis isolates from 2012.

\section{Analysing genetic diversity}

The genetic diversity of the isolates sharing the IbA10G2 genotype was analysed further with samples with the
IbA10G2 genotype that were collected in previous years [7]. A total of 32 samples was analysed with the same set of microsatellite markers, except for marker MS9. Of these, 23 were fully genotyped, 5 were partially genotyped and 4 were negative. The estimated size of the alleles at each locus were identical to those found for the IbA10G2 samples collected in 2012.

The mean genetic diversity of the $C$. hominis isolates from 2012 was estimated to be 0.022 (95 \% highest posterior density $(\mathrm{HPD})=0.015-0.029)$, whereas it was 0.015 (95\% HPD $=0.010-0.024)$ for the C. hominis isolates collected during the previous epidemiological investigation. The estimated probability that the genetic diversity of $C$. hominis increased in 2012 compared to the previous years was 0.88 , thus the probability that it decreased or remained constant was 0.12 .

\section{Discussion}

In August 2012, an unusual rise of the number of cryptosporidiosis cases occurred in The Netherlands. At the early stage, it was unclear whether such increase was due to the known seasonality of this infection, i.e. to the usual summer peak, or to an ongoing, undetected outbreak. In the same period, an increase in cases was also noticed in Germany and in the UK. As in the Netherlands, the dominant species in the UK was $C$. 
hominis. The epidemiological study into the cause of the increase did not reveal a clear common source [23]. Additionally, it seemed unlikely that a single contamination source, such as a single type of food, could be responsible for the observed increase not only across The Netherlands, but also in Germany and the UK.

To gain additional insights, a genetic characterization of isolates from The Netherlands was undertaken. This revealed that a specific GP60 genotype was clearly predominating, although other GP60 genotypes were also found, adding up to $19 \%$ of the total. The initial goal was to refine the case definition for the epidemiological survey in order to strengthen the data leading to a cause. Statistically significant risk factors, however, were not found [23]. Nevertheless, the finding that $81 \%$ of all cases were caused by the IbA10G2 genotype may be considered as compatible with a major single contamination source. We, therefore, analysed some of the IbA10G2 samples with microsatellite markers. The analysis of six microsatellite markers revealed no length polymorphism among samples from 2012 or from previous years, suggesting that the IbA10G2 genotype from the Netherlands represents a genetically homogeneous strain.

Does the high percentage of the IbA10G2 genotype in Dutch patients simply reflect its overwhelming presence in the environment? We do not know, but we do know from previous data from The Netherlands and the neighbouring region of East Flanders, that this has been the most commonly identified $C$. hominis genotype in the last years. Wielinga et al. 7 presented GP60 genotype data on Cryptosporidium spp. found in clinical samples from The Netherlands between the years 2003 and 2005. The study by Wielinga et al. was conducted differently from this study. We aimed to type as many samples as possible from patients diagnosed at a large number of laboratories across the country during a limited period of approximately 5 months. On the other hand, Wielinga et al. collected samples from a limited number of MMLs, not covering the entire country, and during several years. Nevertheless, in these samples the IbA10G2 genotype was also the predominant genotype, found in 65 out of the 80 samples from 2003 to 2005. The 2009 study from Belgium also confirms the predominance of the IbA10G2 genotype, although this was less striking with 10 of 24 samples [31].

The mean genetic diversity of the 2012 samples was higher than that of the samples from 2003 to 2005. Although the estimated probability for the increase in the genetic diversity was less than the threshold of 0.95 by 0.07 , the change took place at the same period in which the incidence rate also increased. This is in line with our previous findings that indicated that the genetic diversity of GP60 may be an independent and complementary measure for quantifying disease incidence [32].
In an outbreak situation, where there is only a limited number of genotypes involved in the infection of a large population, one might have expected a short-term decrease in genetic diversity rather than an increase. Detailed genetic analyses of (future) outbreaks of Cryptosporidium spp. may help us to be able to distinguish between upsurges and unapparent outbreaks of cryptosporidiosis.

\section{Conclusions}

In conclusion, the increase in the number of Cryptosporidium-positive patients in 2012 was predominantly caused by $C$. hominis genotype IbA10G2. The similar distribution of Cryptosporidium genotypes was also identified in previous years. Both the mean genetic diversity of $C$. hominis and the number of patients increased compared to the previous years. The conclusion of this finding is that the rise reflects more an overall increase and not a common source outbreak.

\section{Competing interests}

The authors declare that they have no competing interests.

\section{Authors' contributions}

$J H R$ and HS performed sequence analyses and SMC performed additional genotyping. $\mathrm{HS}$ and $\mathrm{KT}$ performed the population genetic analysis. MK set up the real-time PCR and analysed all samples. Study design and sample collection was done by WVP, LMK an JVdG. JH drafted and wrote the final version with major contributions by all other authors. All authors read and approved the final version of the manuscript.

\section{Acknowledgements}

We thank Sietze Brandes, Cecile Dam, Denise Hoek and Nahid Nozari for technical assistance. We also are grateful for the close collaboration with the MML's providing the Cryptosporidium spp. samples.

\section{Author details}

${ }^{1}$ Centre for Infectious Disease Control Netherlands, National Institute for Public Health and the Environment (RIVM), Bilthoven, The Netherlands. ${ }^{2}$ Department of Infectious, Parasitic and Immunomediated Diseases Istituto Superiore di Sanità, Rome, Italy.

Received: 10 July 2015 Accepted: 20 February 2016

Published online: 10 March 2016

\section{References}

1. Nime FA, Burek JD, Page DL, Holscher MA, Yardley JH. Acute enterocolitis in a human being infected with the protozoan Cryptosporidium. Gastroenterology. 1976;70(4):592-8.

2. Xiao L, Fayer R, Ryan U, Upton SJ. Cryptosporidium taxonomy: recent advances and implications for public health. Clin Microbiol Rev. 2004;17(1):72-97.

3. Slapeta J. Cryptosporidiosis and Cryptosporidium species in animals and humans: a thirty colour rainbow? Int J Parasitol. 2013;43(12-13):957-70.

4. Morgan-Ryan UM, Fall A, Ward LA, Hijjawi N, Sulaiman I, Fayer R, Thompson RC, Olson M, Lal A, Xiao L. Cryptosporidium hominis n. sp. (Apicomplexa: Cryptosporidiidae) from Homo sapiens. J Eukaryot Microbiol. 2002;49(6):433-40.

5. Fayer R, Santin M, Macarisin D. Cryptosporidium ubiquitum n. sp. in animals and humans. Vet Parasitol. 2010;172(1-2):23-32.

6. Caccio S, Pinter E, Fantini R, Mezzaroma I, Pozio E. Human infection with Cryptosporidium felis: case report and literature review. Emerg Infect Dis. 2002;8(1):85-6.

7. Wielinga PR, de Vries A, van der Goot TH, Mank T, Mars MH, Kortbeek LM, van der Giessen JW. Molecular epidemiology of Cryptosporidium in humans and cattle in The Netherlands. Int J Parasitol. 2008;38(7):809-17.

8. Lebbad M, Beser J, Insulander M, Karlsson L, Mattsson JG, Svenungsson B, Axen C. Unusual cryptosporidiosis cases in Swedish patients: extended 
molecular characterization of Cryptosporidium viatorum and Cryptosporidium chipmunk genotype I. Parasitology. 2013;140(14):1735-40.

9. Li N, Xiao L, Alderisio K, Elwin K, Cebelinski E, Chalmers R, Santin M, Fayer R, Kvac M, Ryan U et al. Subtyping Cryptosporidium ubiquitum, a zoonotic pathogen emerging in humans. Emerg Infect Dis. 2014;20(2):217-24.

10. Peng MM, Xiao L, Freeman AR, Arrowood MJ, Escalante AA, Weltman AC, Ong CS, Mac Kenzie WR, Lal AA, Beard CB. Genetic polymorphism among Cryptosporidium parvum isolates: evidence of two distinct human transmission cycles. Emerg Infect Dis. 1997;3(4):567-73.

11. Morgan U, Weber R, Xiao L, Sulaiman I, Thompson RC, Ndiritu W, Lal A, Moore A, Deplazes P.. Molecular characterization of Cryptosporidium isolates obtained from human immunodeficiency virus-infected individuals living in Switzerland, Kenya, and the United States. J Clin Microbiol. 2000;38(3):1180-3.

12. Control ECfDPa. Annual epidemiological report Reporting on 2010 surveillance data and 2011 epidemic intelligence data. Stockholm: European Centre for Disease Prevention and Control; 2013. p. 4.

13. McLauchlin J, Amar C, Pedraza-Diaz S, Nichols GL. Molecular epidemiological analysis of Cryptosporidium spp. in the United Kingdom: results of genotyping Cryptosporidium spp. in 1,705 fecal samples from humans and 105 fecal samples from livestock animals. J Clin Microbiol. 2000;38(11):3984-90.

14. Chalmers RM, Elwin K, Thomas AL, Guy EC, Mason B. Long-term Cryptosporidium typing reveals the aetiology and species-specific epidemiology of human cryptosporidiosis in England and Wales, 2000 to 2003. Euro Surveill. 2009; 14(2).

15. Chalmers RM, Smith R, Elwin K, Clifton-Hadley FA, Giles M. Epidemiology of anthroponotic and zoonotic human cryptosporidiosis in England and Wales, 2004-2006. Epidemiol Infect. 2011;139(5):700-712.

16. Sorvillo F, Beall G, Turner PA, Beer VL, Kovacs AA, Kraus P, Masters D, Kerndt PR. Seasonality and factors associated with cryptosporidiosis among individuals with HIV infection. Epidemiol Infect. 1998;121(1):197-204.

17. Snel SJ, Baker MG, Kamalesh V, French N, Learmonth J. A tale of two parasites: the comparative epidemiology of cryptosporidiosis and giardiasis. Epidemiol Infect. 2009;137(11):1641-50.

18. Lange $\mathrm{H}$, Johansen $\mathrm{OH}$, Vold L, Robertson $\mathrm{L}$, Anthonisen IL, Nygard K. Second outbreak of infection with a rare Cryptosporidium parvum genotype in schoolchildren associated with contact with lambs/goat kids at a holiday farm in Norway. Epidemiol Infect. 2014;142(10):2105-13.

19. Gherasim A, Lebbad M, Insulander M, Decraene V, Kling A, Hjertqvist M, Wallensten A. Two geographically separated food-borne outbreaks in Sweden linked by an unusual Cryptosporidium parvum subtype, October 2010. Euro Surveill. 2012;17(46)

20. Artieda J, Basterrechea M, Arriola L, Yague M, Albisua E, Arostegui N, Astigarraga U, Botello R, Manterola JM. Outbreak of cryptosporidiosis in a child day-care centre in Gipuzkoa, Spain, October to December 2011. Euro Surveill. 2012; 17(5).

21. Baldursson S, Karanis P. Waterborne transmission of protozoan parasites: review of worldwide outbreaks - an update 2004-2010. Water Res. 2011; 45(20):6603-14.

22. Waldron LS, Ferrari BC, Cheung-Kwok-Sang C, Beggs PJ, Stephens N, Power ML. Molecular epidemiology and spatial distribution of a waterborne cryptosporidiosis outbreak in Australia. Appl Environ Microbiol. 2011;77(21): 7766-71.

23. Fournet N, Deege MP, Urbanus AT, Nichols G, Rosner BM, Chalmers RM, Gorton R, Pollock KG, van der Giessen JW, Wever PC et al. Simultaneous increase of Cryptosporidium infections in the Netherlands, the United Kingdom and Germany in late summer season, 2012. Euro Surveill. 2013;18(2).

24. Hadfield SJ, Robinson G, Elwin K, Chalmers RM. Detection and differentiation of Cryptosporidium spp. in human clinical samples by use of real-time PCR. J Clin Microbiol. 2011;49(3):918-24.

25. Strong WB, Gut J, Nelson RG. Cloning and sequence analysis of a highly polymorphic Cryptosporidium parvum gene encoding a 60-kilodalton glycoprotein and characterization of its 15- and 45-kilodalton zoite surface antigen products. Infect Immun. 2000;68(7):4117-34.

26. Alves M, Xiao L, Sulaiman I, Lal AA, Matos O, Antunes F. Subgenotype analysis of Cryptosporidium isolates from humans, cattle, and zoo ruminants in Portugal. J Clin Microbiol. 2003:41(6):2744-7.

27. Drumo R, Widmer G, Morrison L, Tait A, Grelloni V, D'Avino N, Pozio E, Caccio SM. Evidence of host-associated populations of Cryptosporidium parvum in Italy. Appl Environ Microbiol. 2012;78(10):3523-9.
28. Drummond AJ, Rambaut A. BEAST: Bayesian evolutionary analysis by sampling trees. BMC Evol Biol. 2007;7:214.

29. Katoh K, Misawa K, Kuma K, Miyata T. MAFFT: a novel method for rapid multiple sequence alignment based on fast Fourier transform. Nucleic Acids Res. 2002;30(14):3059-66.

30. Gelman A, Carlin JB, Stern HS, Dunson DB, Vehtari A, Rubin DB. Bayesian Data Analysis, 3rd edn. Boca Raton, Florida, USA: CRC Press; 2013.

31. Geurden T, Levecke B, Caccio SM, Visser A, De Groote G, Casaert S, Vercruysse J, Claerebout E. Multilocus genotyping of Cryptosporidium and Giardia in non-outbreak related cases of diarrhoea in human patients in Belgium. Parasitology. 2009:136(10):1161-8.

32. Takumi K, Caccio SM, van der Giessen J, Xiao L, Sprong H. Hypothesis: Cryptosporidium genetic diversity mirrors national disease notification rate. Parasite Vector. 2015;8:308.

\section{Submit your next manuscript to BioMed Central and we will help you at every step:}

- We accept pre-submission inquiries

- Our selector tool helps you to find the most relevant journal

- We provide round the clock customer support

- Convenient online submission

- Thorough peer review

- Inclusion in PubMed and all major indexing services

- Maximum visibility for your research

Submit your manuscript at www.biomedcentral.com/submit

) Biomed Central 